\title{
X-ray spectra from accretion disks illuminated by protons
}

\author{
B. Deufel, C. P. Dullemond, and H. C. Spruit \\ Max-Planck-Institut für Astrophysik, Karl-Schwarzschildstr. 1, 85740 Garching, Germany \\ Received 31 August 2001 / Accepted 26 February 2002 \begin{abstract}
is either embedded in a magnetically heated accretion disk corona or partly extends into an ion supported torus (or ADAF). We calculate the stationary equilibrium between proton heating, electron thermal conduction and the radiative losses by bremsstrahlung and Compton scattering. A heated surface layer on top of the accretion disk is produced with temperatures between $60-90 \mathrm{keV}$ above a cool layer with temperatures of $0.01 \mathrm{keV}$ (AGN) and $1 \mathrm{keV}$ (galactic black hole candidates). The spectra produced by the surface layer are reminiscent of hard state spectra, but a bit too steep, especially for AGN's. Near the inner edge of the disk, where the optical depth of the disk $\tau \lesssim 1$, we find that the cool component of the disk disappears. Instead, the hot protons from the corona/ADAF heat the disk, on a dynamical time-scale, to temperatures of several $100 \mathrm{keV}$, limited by pair production. This region, here called a "warm disk", could contribute significantly to the hard X-ray spectra and could be important for feeding material into an ADAF.
\end{abstract} \\ Abstract. The X-ray spectrum from a cool accretion disk heated by virialized protons is computed. The cool disk
}

Key words. accretion, accretion disks - black hole physics - X-rays: galaxies - X-rays: stars

\section{Introduction}

The X-ray spectra of galactic black hole candidates $(\mathrm{BH})$ and active galactic nuclei (AGN) are remarkably similar, in spite of their large differences in mass and length scales. The similarities of the observed spectra are evident in their power law shape of energy index $s \approx 1$ in the medium X-ray range, and their high energy cut-off at $E_{\mathrm{c}} \approx 200 \mathrm{keV}$. It is widely accepted that the power law spectra are produced by inverse Compton scattering of soft photons on hot thermal electrons (Shapiro et al. 1976; Sunyaev \& Titarchuk 1980; Pozdnyakov et al. 1983). Such spectra depend on the optical depth and the temperature of the Comptonizing region. In view of the similarities in the observed power laws these two parameters apparently vary only within narrow ranges for different sources.

Another common feature evident in many of the observed spectra is the signature of a fluorescent Fe emission line and a Compton-reflection component. These two components provide a strong indication that the X-ray production region is very close to cold matter in the central regions of BHs or AGNs (e.g. Ross \& Fabian 1993).

The classical accretion disk model (Shakura \& Sunyaev 1973) can not explain the simultaneous presence of cold and hot matter near a compact object. Another mode of accretion was subsequently proposed by

Send offprint requests to: B. Deufel, e-mail: bed@mpa-garching.mpg.de
Shapiro et al. (1976). They showed that there is an optically thin hot disk solution where the plasma is in a twotemperature state, with the ions near their virial temperature in the inner region of the accretion disk. This model could in principle account for the observed Comptonized spectra. In this model the accretion flow is optically thin and radiatively inefficient. It was called an ion supported torus by Rees et al. (1982).

The solution described by Shapiro et al. (1976) turned out to be thermally unstable. Stable accretion is obtained when the advection of internal energy with the flow is taken into account (Narayan \& Yi 1994; Narayan \& Yi 1995a,b); these models are called advection dominated accretion flows (ADAF). Most of the viscously dissipated energy is advected radially with the flow. The protons are near their virial temperature, whereas the electrons are much cooler due to their strong interaction with the radiation field and the low rate at which they can exchange energy with the protons via Coulomb interactions. The flow may in principle coexist with an optically thick, cold accretion disk such that the cold disk partly extends into the hot torus (Esin et al. 1997).

Another possibility for the simultaneous presence of cold and hot matter are accretion disk corona (ADC) models (e.g. Nakamura \& Osaki 1993; Haardt \& Maraschi 1991; Haardt \& Maraschi 1993; Svensson \& Zdziarski 1994; Dove et al. 1997). In these models the cold accretion disk is embedded in a hot corona in a plane-parallel slab 
configuration. A large fraction of the gravitational energy is assumed to be released in the corona by magnetic fields, although the details of the coronal heating process are still unclear. The protons in the corona are hot, whereas the disk beneath is cool $(k T \simeq 1 \mathrm{keV})$, optically thick, geometrically thin and gas pressure supported.

In both the ADAF and the ADC models there is an energy coupling between the cold disk material and the hot tenuous plasma above. Traditionally this interaction was seen in terms of an exchange of radiation (Haardt \& Maraschi 1991, 1993; Haardt et al. 1994, 1997). Here we are interested in the energetic coupling via the exchange of matter. We investigate the penetration of hot protons from the ADC/ADAF into the cool disk, i.e. "ion illumination". The hot protons are stopped in the cold disk due to Coulomb interactions with the ambient electrons, rather than by binary interactions with target ions.

Proton illumination is not restricted to accretion disks. It was applied early in X-ray astronomy to explain spectra of galactic X-ray sources as neutron star surfaces heated by radially infalling ions (Zel'dovich \& Shakura 1969; Alme \& Wilson 1973; Turolla et al. 1994). Recently Deufel et al. (2001) revisited the proton illumination of a neutron star in the context of accretion from an ADAF.

The importance of proton illumination for accretion disks was suggested by Spruit (1997) and Spruit \& Haardt (2000). Detailed Monte Carlo calculations of the Comptonization were presented by Deufel \& Spruit (2000) (henceforth Paper I). For the present work we have improved the treatment of the radiative processes by solving the radiative transfer equation including thermal emission due to bremsstrahlung. Pair production is also taken into account. We further allow for energy redistribution due to thermal electron conduction within the disk. The interaction between the impinging protons and the accretion disk is computed time dependently in a one-dimensional, plane-parallel approximation. The density distribution is found from hydrostatic equilibrium including the force exerted by the decelerating protons.

The cool disk acts as an effective thermalizer for energetic (Comptonized) photons as long as its optical depth is large enough. At lower optical depth, such as occurs near the inner edge of the disk, photon production is less efficient. Therefore we expect different solutions for disks of low optical depth. This is borne out by the results reported in Sect. 5 .

\section{A cool disk extending inside an ion supported flow}

\subsection{Optically thick case}

Most of the physics of the interaction between the hot supported flow and the underlying cool disk is rather independent of the structure of the cool disk, since it acts mainly as a thermalizing surface. The height above the midplane where the hot ion-supported flow and the cold disk interact is important, however, since this determines the gravitational acceleration, and hence the density within the interaction layer. Processes such as photon production by bremsstrahlung depend on the electron density explicitly. To obtain an estimate for the thickness of the cool disk, the amount of energy released in the cool disk must be specified. For this purpose we adopt an $\alpha$-disk prescription as described in Svensson \& Zdziarski (1994). In this model, a fixed fraction $f$ of the gravitational energy release takes place above the disk, and a fraction $1-f$ in the cool disk. For details we refer also to Paper I.

In order to simplify our treatment we assume that the vertical disk structure can be approximated at a given radius by a plane-parallel, one-dimensional geometry, so that the vertical $z$-coordinate is the only dimension relevant to our problem. We find the vertical density distribution from the equation of hydrostatic equilibrium,

$\frac{\mathrm{d} P}{\mathrm{~d} \tau}=\frac{\Omega_{\mathrm{K}}^{2} z}{\kappa}+\frac{\partial P_{\mathrm{p}}}{\partial \tau}(\tau)$,

where $\Omega_{\mathrm{K}}=\left(G M / R^{3}\right)^{1 / 2}$ is the local Kepler angular velocity, $\kappa=\kappa_{\mathrm{es}}=0.40 \mathrm{~cm}^{2} \mathrm{~g}^{-1}$ is the electron scattering opacity and $\tau$ is the electron scattering optical depth. Note that we use $\tau$ here as a coordinate, replacing the vertical height $z$. The first term on the right represents the usual weight of the disk; the second term takes into account the momentum exerted by the deceleration of the incident protons as a function of optical depth. The force $\partial P_{\mathrm{p}} / \partial \tau$ is evaluated by recording the change of velocity $\Delta v_{\mathrm{p}}$ of the incoming protons as a function of optical depth (see Sect. 3.1). The contribution of the radiative pressure is neglected as we study cases well below the Eddington luminosity.

Together with the equation for the vertical coordinate $z$,

$\frac{\mathrm{d} z}{\mathrm{~d} \tau}=\frac{-k}{\mu m_{\mathrm{p}} \kappa} \frac{T(\tau)}{P(\tau)}$,

we integrate Eq. (1) via a fourth order Runge-Kutta method. We set $\mu=\frac{1}{2}$ for the idealized case of an ionized hydrogen atmosphere.

The initial value for the pressure at the top of the layer is set to a small fraction of the coronal pressure, $P_{\text {top }}(\tau=0)=10^{-2} n_{\mathrm{p}} k T_{\text {vir }}$. The geometrical height $z_{\text {top }}$ of this upper boundary layer is not known in advance. We solve the pressure profile by starting with an initial guess for the height of the disk above the mid-plane, $z_{\mathrm{top}}$. We integrate Eq. (1) to the maximum optical depth of our model atmosphere $\tau_{\text {bot }}$ at the vertical height $z_{\text {bot }}$. Usually we set $\tau_{\text {bot }}=3$. At this Thomson optical depth the protons have already lost more than 0.99 of their energy. At $\tau_{\text {bot }}$ pressure balance between the simulated layer and the underlying cool disk requires $P\left(\tau_{\text {bot }}\right)=P\left(z_{\text {bot }}\right)$. We iterate until we find the right value for which this pressure condition at $z_{\text {bot }}$ is fulfilled. Thus we have matched the simulated layer to the underlying cool disk. The temperature of the cool disk and the pressure at the mid-plane are determined as in Paper I. 


\subsection{Moderate optical depth}

Near the inner edge of a cool Shakura-Sunyaev disk at the radial distance $R_{\mathrm{i}}$ from the compact object, the surface density drops to low values. In the limit $H / R \ll 1$ (where $H$ is the disk pressure scale height), the surface density varies as $\left[1-\left(R_{\mathrm{i}} / R\right)^{1 / 2}\right]$. Close to the inner edge of an accretion disk, the assumption of a large optical depth is therefore not valid any more. A certain minimum optical depth is necessary, however, for efficient cooling of the disk by soft photons to be possible. In Sect. 5 we explore cases where the total optical depth of the layer is of order unity or less. The procedure to calculate the hydrostatic balance in such cases is almost the same as in the cases of a surface layer on top of an optically thick disk. But instead of matching the computed layer to an underlying cool disk, we put the bottom of the simulated layer is at $z\left(\tau_{\text {bot }}\right)=0$, the mid-plane of the disk. We are interested in simulating the complete disk, i.e. from the upper surface to the lower surface. We do this by introducing an artificial "mirror" placed at the mid-plane. All relevant physical quantities of the model are reflected at this mirror. Thus we have to compute one half space only instead of simulating the whole vertical disk structure.

\section{Heating and cooling processes of the model}

\subsection{Proton illumination of the accretion disk}

\subsubsection{Coulomb interactions in an ionized plasma}

At the typical energies of the protons incident on the cool disk, the energy loss is mostly by long-range Coulomb interactions with the electrons in the disk (small-angle scattering on the large number of electrons in a Debye sphere). This is opposite to the case of protons with a temperature near that of the plasma in which they move. In the latter case, the equilibration among the protons is faster than between electrons and protons, by a factor of order $\left(m_{\mathrm{p}} / m_{\mathrm{e}}\right)^{1 / 2}$. To see how this apparent contradiction is resolved, consider the basic result for the energy loss of a charged particle moving in a fully ionized, charge-neutral plasma. This was derived by Spitzer (1962) (making use of Chandrasekhar's (1942) earlier results on dynamical friction). Introduce as a measure of distance in the plasma the Thomson optical depth $\tau$, i.e. $\mathrm{d} \tau=\sigma_{\mathrm{T}} n \mathrm{~d} l$, where $\sigma_{\mathrm{T}}=8 \pi e^{4} /\left(3 m_{\mathrm{e}}^{2} c^{4}\right)$ is the Thomson cross section, $n$ the electron density and $l$ the distance. The rate of change of energy $E=m_{\mathrm{p}} v^{2} / 2$ of a proton moving with velocity $v$ in a field of particles with charge $e$ and mass $m_{\mathrm{f}}$ (the "field particles" in Spitzer's nomenclature) is then given by Spitzer's Eq. (5)-(15). In our notation, this can be written in terms of the energy loss length $\tau_{\mathrm{f}}$ for interaction with the field particles $\mathrm{f}$,

$\tau_{\mathrm{f}}^{-1}=\frac{1}{E}\left(\frac{\mathrm{d} E}{\mathrm{~d} \tau}\right)_{\mathrm{f}}=3 \ln \Lambda\left(\frac{m_{\mathrm{e}}}{m_{\mathrm{p}}}\right)^{2}\left(\frac{c}{v}\right)^{4}\left(1+\frac{m_{\mathrm{p}}}{m_{\mathrm{f}}}\right) F\left(x_{\mathrm{f}}\right)$ where $\ln \Lambda$ is the Coulomb logarithm (which is determined by the size of the Debye sphere). Here

$F(x)=\psi(x)-x \psi^{\prime}(x)$,

where $\psi$ is the error function and $\psi^{\prime}$ its derivative, and $x_{\mathrm{f}}=v\left[m_{\mathrm{f}} /(2 k T)\right]^{1 / 2}$ is (up to a numerical factor) the ratio of the incident proton's velocity to the thermal velocity of the field particles. The limiting forms of $F$ are

$$
F(x) \rightarrow x^{3} / 3 \quad(x \rightarrow 0), \quad F \rightarrow 1 \quad(x \rightarrow \infty) .
$$

We can evaluate (3) under the assumption that the field particles that are most relevant for the energy loss are the protons or the electrons, respectively, and compare the loss lengths. If the incident proton has velocity comparable with the thermal velocity of the field protons, we have $x_{\mathrm{p}} \sim 1$, and $x_{\mathrm{e}}=\left(m_{\mathrm{e}} / m_{\mathrm{p}}\right)^{1 / 2} x_{\mathrm{p}} \ll 1 . F\left(x_{\mathrm{p}}\right)$ is then of order unity and $F\left(x_{\mathrm{e}}\right) \approx x_{\mathrm{e}}^{3} / 3$. Setting $\mathrm{f}=\mathrm{e}$ respectively $\mathrm{f}=\mathrm{p}$ in (3) and taking the ratio, we have

$\frac{\tau_{\mathrm{e}}}{\tau_{\mathrm{p}}} \approx\left(\frac{m_{\mathrm{p}}}{m_{\mathrm{e}}}\right)^{1 / 2}$

The loss length for interaction with the electrons is thus much longer than for interaction with the protons, and the interaction with electrons can be neglected. This well known result is the relevant limit for the relaxation of a proton distribution in a plasma that is not too far from its thermal equilibrium.

For incoming protons of high energy, however, the result is different because $x_{\mathrm{e}}$ is not sufficiently small any more. In the high- $v$ limit, $F\left(x_{\mathrm{e}}\right)=F\left(x_{\mathrm{p}}\right)=1$, and one has

$\frac{\tau_{\mathrm{e}}}{\tau_{\mathrm{p}}} \approx 2 \frac{m_{\mathrm{e}}}{m_{\mathrm{p}}}=10^{-3}$.

In this limit, the energy loss is thus predominantly to the electrons. A related case is that of the ionization losses of fast particles in neutral matter (for references see Ryter et al. 1970). The case of an ionized plasma is simpler, since the electrons are not bound in atoms. The change from proton-dominated loss to electron-dominated loss takes place at an intermediate velocity $v_{\mathrm{c}}$, at which $F\left(x_{\mathrm{p}}\right) \approx 1$ but $x_{\mathrm{e}}$ still small, so that $F\left(x_{\mathrm{e}}\right) \approx x_{\mathrm{e}}^{3} / 3$. Equating $\tau_{\mathrm{e}}$ and $\tau_{\mathrm{p}}$ then yields

$x_{\mathrm{e}, \mathrm{c}} \approx\left(\frac{m_{\mathrm{e}}}{m_{\mathrm{p}}}\right)^{1 / 3}$

or

$\frac{E_{\mathrm{c}}}{k T} \approx\left(\frac{m_{\mathrm{p}}}{m_{\mathrm{e}}}\right)^{1 / 3} \approx 12$.

For the electron temperatures we encounter in our models, $T \sim 100 \mathrm{keV}$, energy loss to the field protons can thus be neglected for incoming protons with energy $E \gtrsim 1 \mathrm{MeV}$. This is the case in all calculations presented here. 


\subsubsection{Corrections at high and low energies}

Spitzer's treatment is non-relativistic, while virialized ADAF protons near the hole can reach sub-relativistic temperatures. A fully relativistic treatment of the Coulomb interactions in a plasma has been given by Stepney \& Guilbert (1983). We have compared the classical treatment according to Spitzer's theory with this relativistic result in Deufel et al. (2001), and found it to be accurate to better than $5 \%$ for proton temperatures $<100 \mathrm{MeV}$. The classical approximation in Spitzer's analysis therefore does not introduce a significant error for the problem considered here.

For high energies, the Coulomb energy loss becomes so small that loss by direct nuclear collisions becomes competitive. This happens (cf. Stepney \& Guilbert 1983) at $E \gtrsim 300 \mathrm{MeV}$, an energy that is not reached by virialized protons except in the tail of their distribution. We ignore these direct nuclear collisions. Note, however, that a gradual nuclear processing by such collisions can be important (Aharonian \& Sunyaev 1984), in particular for the production of the lithium. The lithium overabundances seen in the companions of LMXB (Martín et al. 1994a), may in fact be a characteristic signature of the interaction of an ADAF and a disk described here (Martín et al. 1994b; Spruit 1997).

As the protons slow down, they eventually equilibrate with the field protons. This last part of the process is not accurately described by the energy loss formula (3). In addition to the simple energy loss of a particle moving on a straight path through the plasma, one has to take into account the random drift in direction and energy resulting from the interaction with the fluctuating electric field in the plasma. This drift can be ignored to first order (end of Sect. 5.2 in Spitzer 1962), but takes over in the final process of equilibration with the plasma. This last phase involves negligible energy transfer compared with the initial energy of the protons in the present calculations, and can be ignored here.

\subsubsection{Charge balance}

The protons penetrating into the disk imply a current that has to be balanced by a "return current". As in all such situations, this return current results from the electric field that builds up due to the proton current. This field drives a flow of electrons from the ADAF to the disk which maintains the charge balance. Since the electron density in the disk is high, the return current does not involve a high field strength.

\subsubsection{Calculations}

The numerical method used to calculate the electron heating rates of the accretion disk due to proton illumination is described in detail in Paper I; here we give a short overview.
We follow the evolution of an initially Maxwellian distribution of protons, which we place above the cool accretion disk, as the protons penetrate through the disk atmosphere. For the temperature of the incident protons in our model we take the local virial temperature

$T_{\mathrm{p}}=T_{\text {vir }}=\frac{G M m_{\mathrm{p}}}{3 k_{\mathrm{B}} R} \approx \frac{156}{r} \mathrm{MeV}$,

where $G$ is the gravitational constant, $M$ the mass of the black hole, $m_{\mathrm{p}}$ the proton mass, $R$ the radius from the central object and $k_{\mathrm{B}}$ is the Boltzmann constant. $r$ is the dimensionless radius, $r=R / R_{\mathrm{S}}$, where $R_{\mathrm{S}}=2 G M / c^{2}$ is the Schwarzschild radius.

The energy loss of the incoming protons is computed from (3), with $\mathrm{f}=$ e. Denote by $\tau$ the Thomson optical depth measured vertically into the layer, from its top. The rate of change of energy with depth for a proton at depth $\tau$ moving at an angle $\theta$ with respect to the vertical is then

$\frac{1}{E} \frac{\mathrm{d} E}{\mathrm{~d} \tau}=\frac{3 \ln \Lambda}{\cos \theta} \frac{m_{\mathrm{e}}}{m_{\mathrm{p}}}\left(\frac{c}{v}\right)^{4}\left[\psi(x)-x \psi^{\prime}(x)\right]$,

with

$x=x_{\mathrm{e}}=v\left(\frac{m_{\mathrm{e}}}{2 k T}\right)^{1 / 2}$,

while $T(\tau)$ is the depth-dependent temperature of the layer, and $v$ the depth and angle-dependent velocity of the incident proton. The angular distribution of the protons is discretized in 50 equidistant points. The Debye-length in the plasma, measured in Thomson optical depths, is quite small so that temperature variations over a Debye-length can be ignored and the local energy loss rate at any depth is adequately described by (11).

The energy flux of the incident flux $q_{\mathrm{p}}$ is proportional to the density in the ADAF, which depends on its accretion rate and viscosity. Instead of using detailed models we parameterize these dependences by scaling the energy flux of the incident protons with the local energy dissipation rate in the ADAF. Using the thin-disk expression for this dissipation rate, we set

$q_{\mathrm{p}}=f Q(R)=f \cdot \frac{3 G M \dot{M}}{8 \pi R^{3}} \cdot J(R)$,

where $f$ is an adjustable numerical factor of order unity and $J(R)=1-\left(R_{\mathrm{i}} / R\right)^{1 / 2}$. This scaling of the proton flux is also useful if one has a coronal model in mind instead of an ADAF. The factor $f$ then is interpreted as the fraction of the viscous energy release that is dissipated in the corona.

We follow the protons from the corona numerically through the disk atmosphere and record the energy loss as a function of optical depth $\tau$ according to Eq. (11). This yields the local time dependent heating rate $\Lambda_{\mathrm{P}}(\tau)$.

\subsection{Radiative transfer}

We treat the radiative transfer by solving the radiative transfer equation

$\mu \frac{\mathrm{d} I_{\mu, \nu}}{\mathrm{d} z}=j_{\nu}^{f f}+j_{\mu, \nu}^{\mathrm{c}}-\left(\alpha_{\nu}^{f f}+\alpha_{\nu}^{\mathrm{c}}\right) I_{\mu, \nu}$ 
where $I_{\mu, \nu}$ is the intensity as a function of frequency $\nu$ and photon angle $\mu=\cos (\theta), \alpha_{\nu}^{f f}$ is the bremsstrahlung absorption coefficient, $\alpha_{\nu}^{\mathrm{c}}$ the Compton extinction coefficient, $j_{\nu}^{f f}$ the bremsstrahlung emissivity and $j_{\mu, \nu}^{\mathrm{c}}$ the Compton emissivity. The method for solving the radiative transfer equation is described in Deufel et al. (2001). From the solution of the radiative transfer equation we obtain the radiative cooling rates $\Lambda_{\mathrm{rad}}(\tau)$.

\subsection{Pair processes}

As discussed below, the cases of moderate optical depth can become rather hot so that pair processes have to be included. We include photon-photon pair production $\left(\gamma \gamma \rightarrow \mathrm{e}^{+} \mathrm{e}^{-}\right)$as well as pair production due to ee collisions $\left(\mathrm{ee} \rightarrow \mathrm{eee}^{+} \mathrm{e}^{-}\right)$in a steady state, i.e. the pair production rate equals the pair annihilation rate.

The photon-photon pair production rate can be expressed as a quadruple integral over the dimensionless photon energies $x_{1}=h \nu_{1} / m_{\mathrm{e}} c^{2}, x_{2}=h \nu_{2} / m_{\mathrm{e}} c^{2}$ and photon angles $\mu_{1}, \mu_{2}$, respectively (cf. Zane et al. 1995):

$R_{\gamma \gamma}=\frac{4 \pi}{h^{2} c} \int \mathrm{d} x_{1} \mathrm{~d} x_{2} \mathrm{~d} \mu_{1} \mathrm{~d} \mu_{2} \frac{I_{\mu_{1}, \nu_{1}} I_{\mu_{2}, \nu_{2}}}{x_{1}^{2} x_{2}^{2}} F\left(x_{+}, x_{-}\right)$,

where $F\left(x_{+}, x_{-}\right)$is defined as

$F\left(x_{+}, x_{-}\right)=\int_{x_{-}}^{x_{+}} \frac{\sigma(x) x^{3} \mathrm{~d} x}{\sqrt{\left(x_{+}^{2}-x^{2}\right)\left(x^{2}-x_{-}^{2}\right)}}$,

with $x_{ \pm}^{2} \equiv x_{1} x_{2}\left[1-\cos \left(\theta_{1} \pm \theta_{2}\right)\right] / 2$ (Stepney \& Guilbert 1983). The pair cross-section $\sigma(x)$ is (Jauch \& Rohrlich 1976)

$\sigma(x)=\frac{1}{2}\left(1-\beta^{2}\right)\left[\left(3-\beta^{4}\right) \log \left(\frac{1+\beta}{1-\beta}\right)-2 \beta\left(2-\beta^{2}\right)\right]$,

with $\beta \equiv \sqrt{x^{2}-1} / x$.

In our models we have simplified the above integral by replacing the intensities by their corresponding meanintensities: $I_{\mu_{1}, \nu_{1}} \rightarrow J_{\nu_{1}}$ and $I_{\mu_{2}, \nu_{2}} \rightarrow J_{\nu_{2}}$. For any given value of $y \equiv x_{1} x_{2}$ we can now evaluate the function $G(y) \equiv \int F\left(x_{+}, x_{-}\right) \mathrm{d} \mu_{1} \mathrm{~d} \mu_{2}$, which is independent of the radiation field, and which can be tabulated and stored beforehand. The pair production rate now reduces to

$R_{\gamma \gamma} \approx \int G\left(x_{1} x_{2}\right) J_{\nu_{1}} J_{\nu_{2}} \mathrm{~d} x_{1} \mathrm{~d} x_{2}$.

Thus the quadruple integral is reduced to a double integral, which is much faster to evaluate. An accurate evaluation of this integral can be made by writing $\mathrm{d} x_{1} \mathrm{~d} x_{2}=$ $x_{1} x_{2} \mathrm{~d} \log \left(\sqrt{x_{1} x_{2}}\right) \mathrm{d} \log \left(x_{1} / x_{2}\right)$, since the function $G\left(x_{1} x_{2}\right)$ is independent of $x_{1} / x_{2}$.

The pair production rate due to electron-electron collisions is e.g. given in Svensson (1982) as

$\dot{n}_{+}^{\mathrm{ee}}=\left(n_{+}+n_{-}\right)^{2} c r_{\mathrm{e}}^{2} P_{\mathrm{ee}}(\theta)$

where we have introduced $\theta=k T_{\mathrm{e}} / m_{\mathrm{e}} c^{2}$ and

$P_{\mathrm{ee}}=\frac{1}{2} \alpha_{f}^{2} \frac{28}{27 \pi}(2 \ln 2 \theta)^{3}$.
Here $\alpha_{f}$ is the fine structure constant. This production rate is only included for $\theta>0.6$.

The pair annihilation rate is also given by Svensson (1982) as

$\dot{n}_{+}^{\mathrm{an}}=n_{+} n_{-} c r_{\mathrm{e}}^{2} A(\theta)$,

where

$A(\theta)=\frac{\pi}{1+2 \theta^{2} / \ln (2 \eta \theta+1.3)}$,

$\eta \simeq 0.56146$. Now we can set up the pair balance equation in the stationary case,

$R_{\gamma \gamma}+n^{2} c r_{\mathrm{e}}^{2}\left[(2 z+1)^{2} P(\theta)-z(1+z) A(\theta)\right]=0$.

We have used the charge neutrality condition $n_{-}=n_{+}+n$ and $z=n_{+} / n$ is the ratio of the pair number density to the proton number density $n$. Equation (23) is a quadratic equation for the pair fraction $z$. The positive root of this equation is

$z(\tau)=\frac{1}{2}\left[-1+\frac{\sqrt{A(\theta) c n^{2} r_{\mathrm{e}}^{2}+4 R_{\gamma \gamma}}}{n r_{\mathrm{e}} \sqrt{c} \sqrt{A(\theta)-4 P(\theta)}}\right]$.

The additional electrons from pair production serve as further scattering partners for the Coulomb collisions and the Compton scattering. Effects due to electron-positron bremsstrahlung are not included nor is a treatment of the annihilation line.

\subsection{Energy balance from heating and cooling}

We begin our computation with an isothermal temperature profile in hydrostatic equilibrium according to Sect. 2. The initial temperature throughout the layer is $T_{\mathrm{d}}$ (see Paper I) for the thick disks and we set $T_{\mathrm{e}}=1 \mathrm{keV}$ for disks with moderate optical depths. The solutions do not depend on the initial temperature profile. First we calculate the heating rates $\Lambda_{\mathrm{p}}^{+}(\tau)$ from the Coulomb interactions and the cooling rates $\Lambda_{\text {rad }}^{-}(\tau)$ due to the radiative processes bremsstrahlung and multiple Compton scattering. Additionally the energy redistribution due to electron thermal conductivity is included using Spitzer's classical value, as in Deufel et al. (2001). This process adds the contribution $\Lambda_{\text {cond }}(\tau)$ to the energy balance. The validity of classical electron conduction was checked by evaluating the electron mean free path $\lambda$, which turns out to be of the order $10^{2} \mathrm{~cm}$ or less in the black hole candidate cases shown in Fig. 1. This is much smaller than the temperature gradient length (more than $10^{4} \mathrm{~cm}$ ), so the condition for validity of the electron formula used is amply satisfied. The same holds for the AGN cases.

The equilibrium is computed following the time evolution of the layer until a balance between the heating and cooling processes is obtained. In the optically thick models, the time scales for approaching equilibrium turn out to be a sharp function of depth in the model. To deal with this, an adaptive time stepping process is used in which the time step depends on both time and depth in 
the model. Stability of this process was obtained by scaling this step with the shortest of the energy exchange time scales associated with the contributing heating and cooling processes. With this procedure the time evolution of the model is not realistic, but the final equilibrium obtained is. For the cases with moderate optical depth we use a depth-independent time step. In these cases, the time evolution of the model is also realistic.

The total change of enthalpy per time step as function of optical depth can then be expressed by

$$
\frac{\Delta w(\tau)}{\Delta t}=\rho c_{\mathrm{p}} \frac{\Delta T(\tau)}{\Delta t}=\Lambda_{\mathrm{p}}^{+}(\tau)+\Lambda_{\mathrm{rad}}^{-}(\tau)+\Lambda_{\mathrm{cond}}(\tau)
$$

where $c_{\mathrm{p}}$ is the specific heat at constant pressure.

Now the change of temperature as a function of optical depth can be calculated. With the new temperature profile the hydrostatic structure is updated according to Sect. 2. We follow the simulation until the Coulomb heating is balanced by the radiative cooling and energy redistribution due to electron conductivity. The energy balance of all our computations is better than $10 \%$.

\section{Results of the model computations}

\subsection{Proton illumination of cool disks in $B H$ and $A G N$}

For the thick disks we use the same model parameters as in paper I, i.e. the accretion luminosity $L=0.1 L_{\mathrm{Edd}}$, the viscosity parameter $\alpha=0.1$ and the fraction of the energy released in the virialized atmosphere $f=0.95$. The mass of the galactic $\mathrm{BH}$ is $M_{\mathrm{BH}}=8 M_{\odot}$ and the mass of the $\mathrm{AGN}$ is $M_{\mathrm{AGN}}=8 \times 10^{6} M_{\odot}$.

Figure 1 shows the solution at $r=10$ for a proton energy flux of $F_{\mathrm{p}}=8.1 \times 10^{21} \mathrm{erg} \mathrm{cm}^{-2} \mathrm{~s}^{-1}, T_{\mathrm{p}}=15.6 \mathrm{MeV}$. The cool disk is separated by a sharp temperature front from a hot part at an optical depth of order unity. This step like temperature profile is much more pronounced compared to the results of Paper I. The temperature of the hot part is $T_{\mathrm{e}} \approx 60 \mathrm{keV}$. The hot layer acts as an effective Comptonizing region and a hard spectrum is emitted. The power law index of the $\mathrm{BH}$ spectra in $E F(E)$ is $s \approx 0.2$. At the soft end of the spectrum there is an excess of soft photons with respect to a blackbody with equivalent energy flux. This excess is due to a "reverse photosphere effect", as explained in Deufel et al. (2001).

The upper right panel of Fig. 1 shows the heating and cooling rates. In the narrow transition zone $(\Delta \tau \approx 0.1)$ between the hot top and the cool bottom electron conductivity is not negligible. Elsewhere conductivity plays no role for the energy balance.

The geometrical depth of the heated layer is $z_{\text {tot }} \approx$ $6 \times 10^{5} \mathrm{~cm}$. This is small compared to the distance from the compact object $\left(R=2.3 \times 10^{7} \mathrm{~cm}\right)$. Thus a cool disk with a proton heated skin on top of it can still be considered a "thin" disk.

Figure 2 shows the dependence of the emergent spectrum and the temperature profile on the distance from the compact object in the galactic $\mathrm{BH}$ case. We obtain a result comparable to Paper I, i.e. with increasing distance the temperature of the hot part increases somewhat, whereas the optical depth of the heated layer decreases. A discussion of these effects can be found in Paper I.

For distances $R>50 R_{\mathrm{S}}$ from the galactic $\mathrm{BH}$ we could not find heated solutions. With increasing distance from the central object the proton temperature $\left(\propto R^{-1}\right)$ as well as the proton energy flux $\left(\propto R^{-3}\right)$ decrease. In these conditions we find solutions in which the energy supplied by the incident protons is thermalized directly in the cool disk by bremsstrahlung, without forming a hot surface layer. It is likely that a very thin $(\tau \ll 0.01)$ hot layer still forms in these conditions, but we are unable to resolve it with the present method. This is indicated by the existence of thin hot atmosphere solutions at low incident energy flux in the case of protons heating a neutron star surface (Zampieri et al. 1995; Deufel et al. 2001). It probably also occurs in the present case, but since these thin layers at large distances from the hole do not contribute much to the overall spectrum, we have not pursued this further.

Figure 3 shows the dependence of the emergent spectrum and the temperature profile on the distance from the compact object in the AGN case. We obtain slightly different solutions compared to our results in Paper I. The optical depth of the heated layer is smaller $(\tau \lesssim 0.5)$, the temperature of the hot part is $T_{\mathrm{e}} \approx 70-90 \mathrm{keV}$. Again a steep temperature jump separates it from the cool disk underneath. Due to the smaller optical depth of the heated layer Comptonization is not as efficient. A significant fraction of the incident ions passes through the hot layer and thermalizes in the cool disk underneath. This increases the soft photon flux relative to the Comptonized flux, and produces a steeper spectrum. The index of the AGN spectra in $E F(E)$ is $s \approx 0.6$. Our model does not produce heated solutions at distances $R>20 R_{\mathrm{S}}$ from the compact object, though heated atmospheres might exist at very small optical depths (see note above).

\section{Proton illumination of disks with moderate optical depth}

The temporal evolution of an initially cool $\left(T_{\mathrm{e}}=1 \mathrm{keV}\right)$, thin disk with an optical depth $\tau_{1 / 2}=1.5$ (measured from the surface to the mid-plane) at $r=10$ for the galactic black hole candidate $(\mathrm{BHC})$ case is shown in Fig. 5. The energy flux from the virialized protons is $F_{\mathrm{p}}=8.1 \times 10^{21} \mathrm{erg} \mathrm{cm}^{-2} \mathrm{~s}^{-1}$. In the beginning the temperature of the top layers increases due to the impinging hot protons, whereas the mid-plane region cools due to bremsstrahlung (stage 0-2, Fig. 5). As the top layers are heated, the stopping power of the plasma decreases and the protons penetrate deeper into the disk. Eventually hot protons reach the mid-plane region and proton heating overcomes bremsstrahlung cooling even there [3]. At this stage no effective cooling mechanism is present and the temperature continues to rise everywhere in the disk [4-5]. At temperatures $k T \gtrsim 200 \mathrm{keV}$ pair production becomes more and more important. The extra electrons serve 


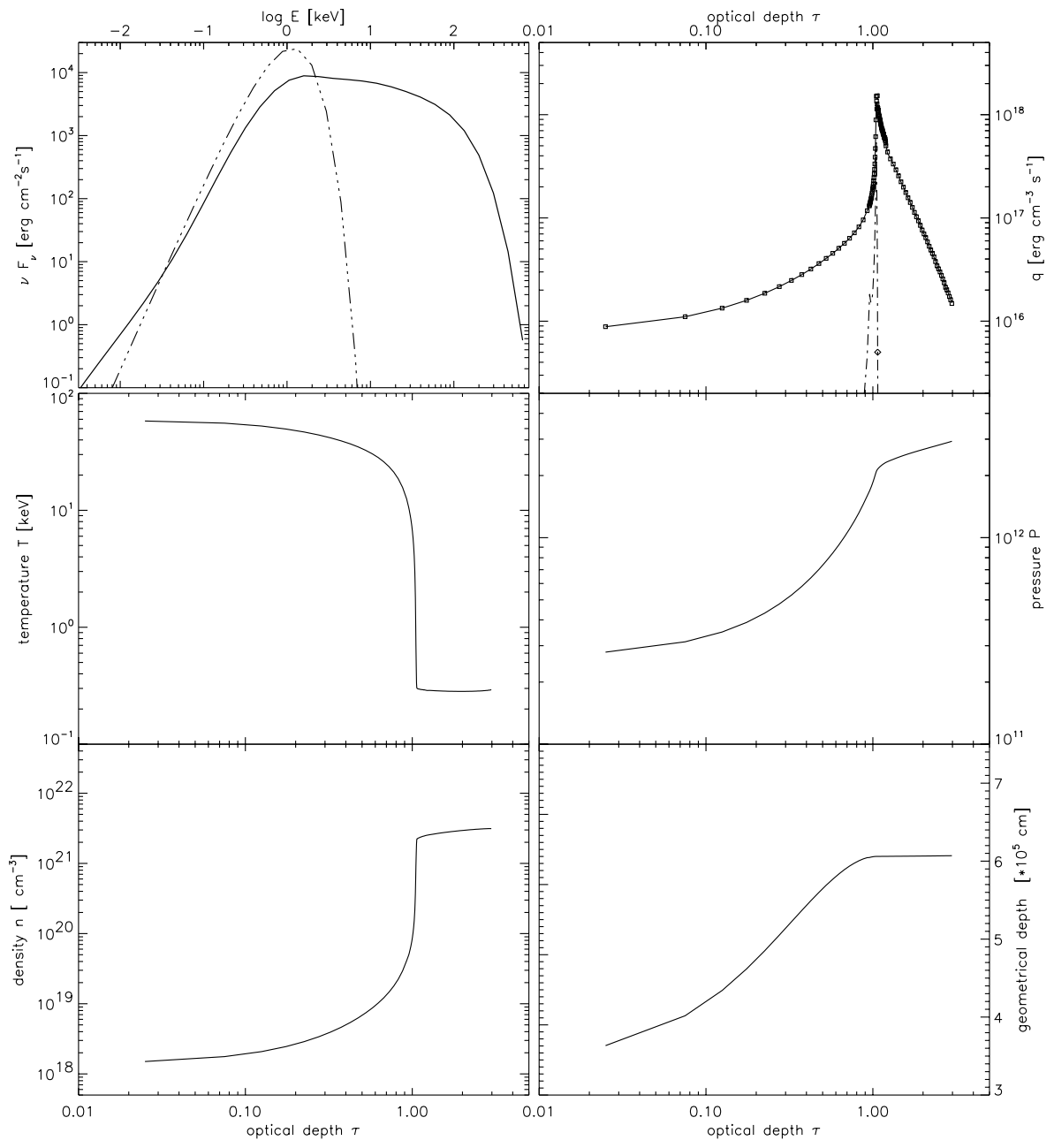

Fig. 1. X-ray spectra of proton- illuminated optically thick cool disks. Upper left panel: emergent model spectrum (solid line) and blackbody with temperature according to proton energy flux (dotted-dashed line); upper right panel: combined heating rates from proton heating and electron thermal conductivity (solid line) and radiative cooling rates due to Comptonization and bremsstrahlung (squares) - the dotted line shows the rates from electron conductivity alone; lower panels show from left to right and from top to bottom the electron temperature $T_{\mathrm{e}}$, pressure $P$, electron density $n_{\mathrm{e}}$ and the geometrical depth of the layer $z / R$, for a solution with $F_{\mathrm{p}}=8.1 \times 10^{21} \mathrm{erg} \mathrm{cm}^{-2} \mathrm{~s}^{-1}$, $T_{\mathrm{p}}=15 \mathrm{MeV}, M=8 M_{\odot}, R=10 R_{\mathrm{g}}$.
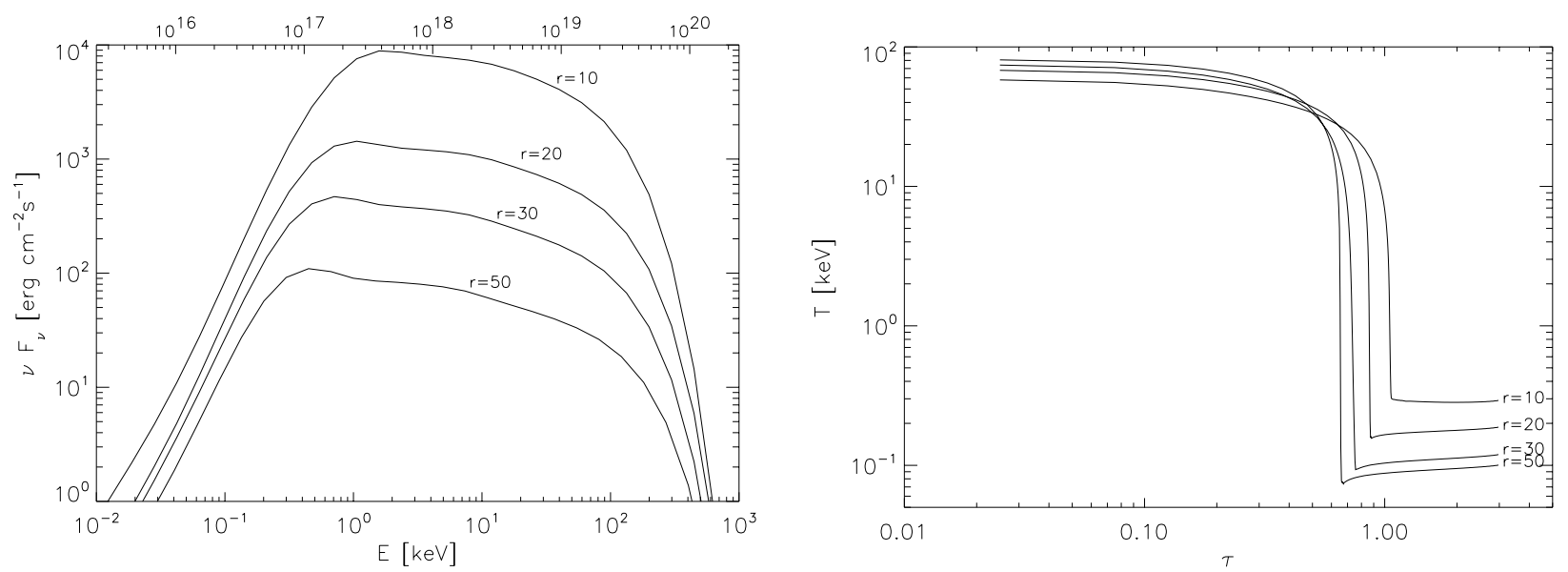

Fig. 2. Dependence of the emergent model spectra and the temperature profiles on the distance from a galactic BH with $M=8 M_{\odot}$. The surface temperature of the of the disk increases slightly with distance, whereas the optical depth of the hot surface layer decreases.

as additional scattering partners for the Coulomb collisions with the protons and the photons of the radiation field. Thus pair production limits the maximum attainable temperatures, and the disk adjusts to a new equilibrium state [6] at a temperatures $T_{\mathrm{e}} \simeq 400 \mathrm{keV}$. The dashed line in Fig. 5 shows the temperature profile after one dynamical time-scale at that radius, $t_{\mathrm{d}}=1 / \Omega_{\mathrm{K}} \simeq 3.5 \times 10^{-3} \mathrm{~s}$.
Figure 4 shows an overview of that solution. The pair number density $z$ at the top of slab reaches $z \approx 50 \%$ of the proton number density and drops off at the midplane to $z \approx 1 \%$. The spectrum of such a thin proton heated disk peaks at $\approx 1000 \mathrm{keV}$. We refer to those disks as warm disks. They are still considerably cooler than the local virial temperature. 

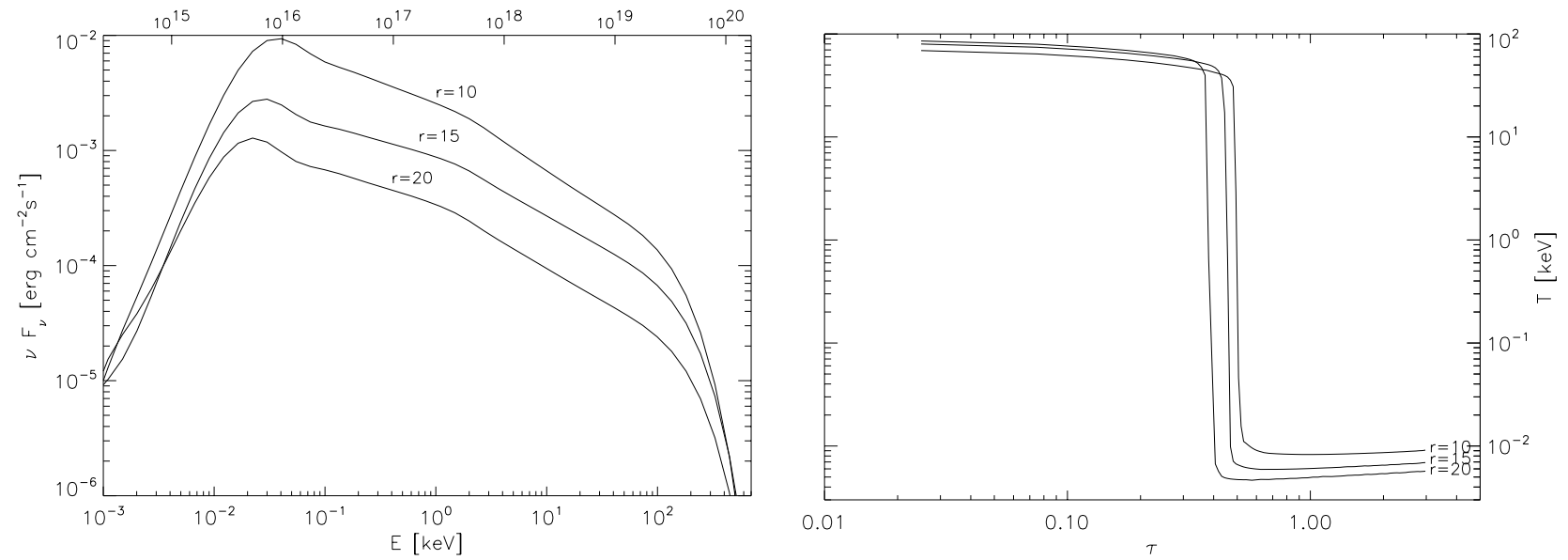

Fig. 3. Dependence of the emergent model spectra and the temperature profiles on the distance from the hole in the AGN case with $M=8 \times 10^{6} M_{\odot}$. The optical depth of the hot part is much smaller than in the galactic BH cases.

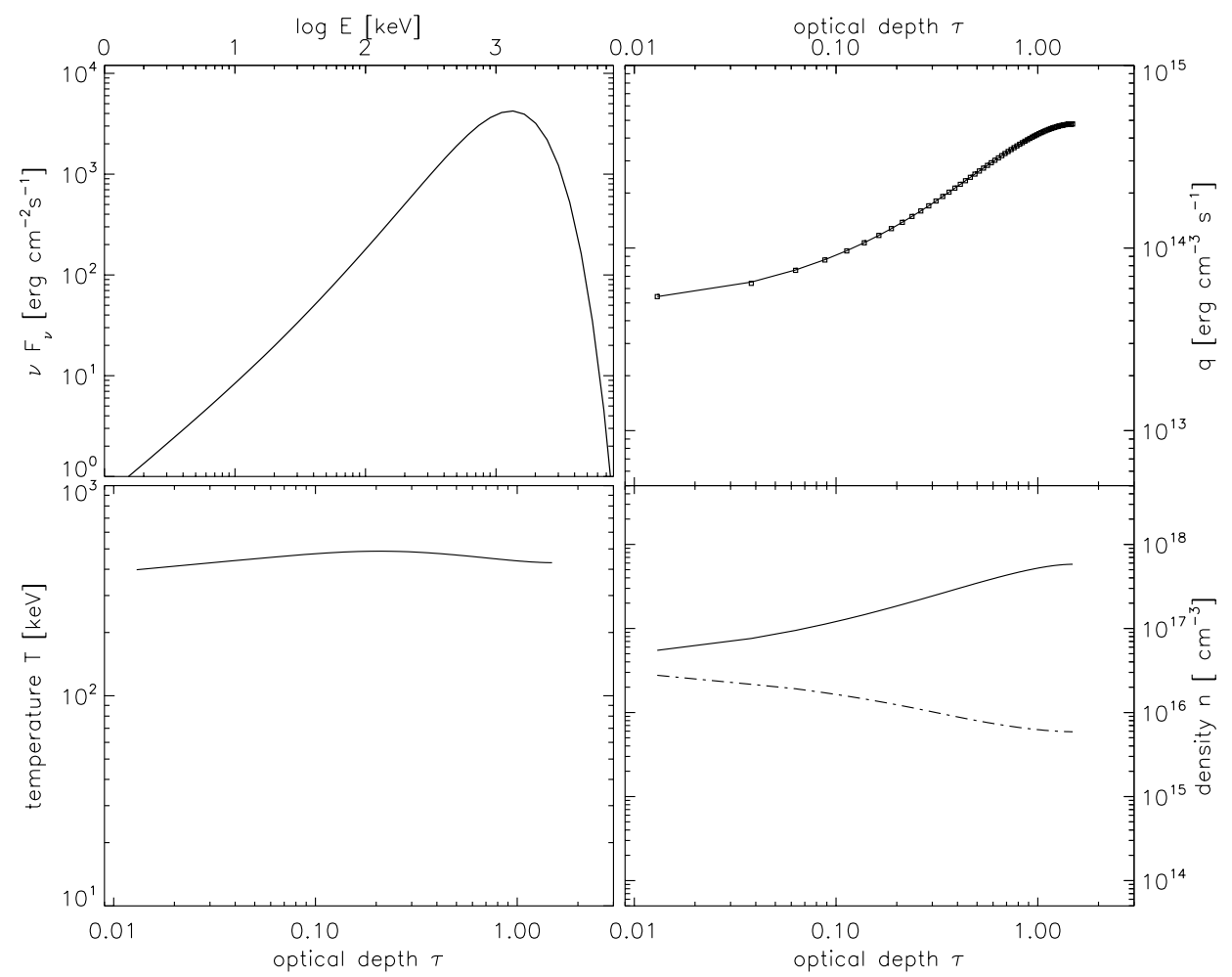

Fig. 4. A "warm disk", of optical depth 1.5, externally heated by ion illumination. Upper left panel: emergent model spectrum; upper right panel: heating rates from proton heating (solid line) and combined radiative cooling rates from Comptonization, bremsstrahlung and pair processes (squares); lower left panel: electron temperature $T_{\mathrm{e}}$; lower right panel: proton number density (solid line) of the layer and pair number density (dashed line).
Figure 6 shows the dependence of the equilibrium temperature profile on the initial optical depth of the layer and the distance from the central object. With increasing distance the proton energy flux as well as the proton penetration depth decreases. A cool disk can therefore be transferred into the warm state only within a certain distance from the $\mathrm{BH}$. If the hot protons do not reach the mid-plane anymore, a cool interior can be maintained which looses its energy very efficiently via bremsstrahlung, as the hot layers above are optically thin. Our model computations show that at $r=15$ a thin disk can maintain a cool interior for $\tau_{1 / 2}=1.5$ whereas at $r=10$ a disk with $\tau_{1 / 2}=1.5$ switches into the warm state.

The temperature of the warm state also depends on both the distance and the overall optical depth of the layer. For $\tau_{1 / 2}=1$ and $r=10$ our model predicts a temperature of $\approx 1 \mathrm{MeV}$. At such temperatures our classical proton heating formalism starts deviating from the correct relativistic expression. The classical treatment underestimates the proton-electron heating rates at high temperatures (Deufel et al. 2001). But further pair processes and radiative cooling terms should also be included (see below), which again limits the maximum temperatures.

The transition from a cool disk to the warm state also takes place in AGNs. For the above AGN parameters (see Sect. 4.1) we find the transition to occur for $\tau_{1 / 2} \lesssim 0.2$ at $r \lesssim 10$. The temperatures of the warm state are in the $\mathrm{MeV}$ range. At such temperatures $(\theta>1)$ our treatment of the pair processes and the radiation field needs more scrutiny. Further pair production processes should 


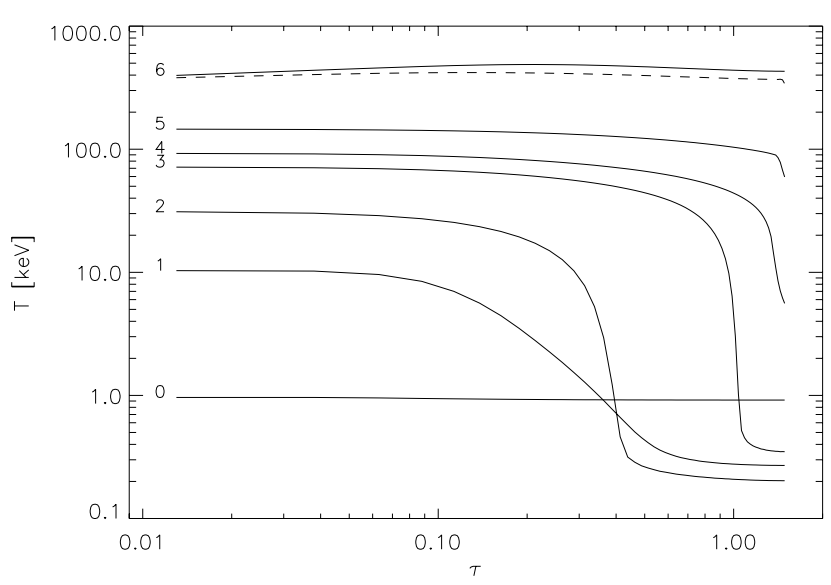

Fig. 5. Evolution from a cool disk to a warm disk by exposure to virialized protons at $r=10$. Initial temperature $T_{\mathrm{e}}=1 \mathrm{keV}$ The numbers at the lines denote the sequential stages, referred to in the text. The final stage [6] shows an equilibrium at $T_{\mathrm{e}} \approx$ $400 \mathrm{keV}$. The dashed line shows the temperature profile after one dynamical time-scale.

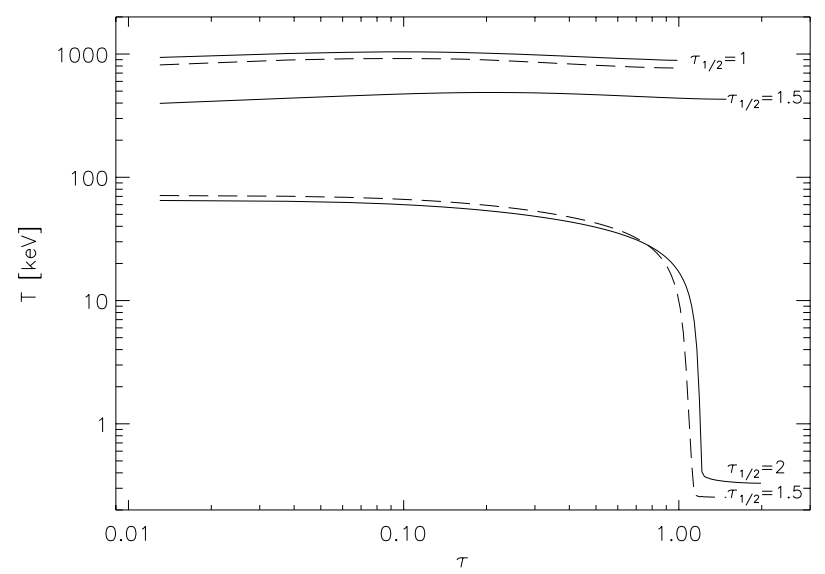

Fig. 6. Equilibrium temperature profiles of warm disks exposed to virialized protons at $r=10$ (solid line) and $r=$ 15 (dashed line) with different initial optical depths $\tau_{1 / 2}=$ $2,1.5,1$. for $r=10$ and $\tau_{1 / 2}=1.5,1$ for $r=15$.

be included $\left(\gamma \mathrm{e} \rightarrow \mathrm{ee}^{+} \mathrm{e}^{-}\right.$, ep $\left.\rightarrow \mathrm{epe}^{+} \mathrm{e}^{-}\right)$as well as additional radiative cooling terms (bremsstrahlung from $\mathrm{e}^{+} \mathrm{e}^{-}$ and $\mathrm{e}^{ \pm} \mathrm{e}^{ \pm}$collisions). These will further limit the maximum attainable temperatures. Therefore we do not think that temperatures of several $\mathrm{MeV}$ found in our simulation of the warm state in AGNs are realistic. The important result of our investigation is that the transition is not only restricted to the BHCs but also takes place in AGNs. But compared to the BHCs the transition in the AGNs occurs in a more narrow zone around the $\mathrm{BH}$ (in terms of Schwarzschild radii) and the vertical extend (in terms of Thomson optical depths) is smaller.

\section{Discussion and conclusions}

We have investigated a model for the production of a hard spectral component $(h \nu \gg 1 \mathrm{keV})$ from a cool disk around a compact object. The model assumes that a standard cool accretion disk is surrounded by a hot atmosphere. This atmosphere can either be due to an ion supported accretion flow (ADAF) which partially overlaps in radial extent with the cool disk or to a hot corona which is e.g. magnetically heated (e.g. di Matteo et al. 1999). For the actual calculations presented here, we have used parameters representative for ADAFs. Fast protons from this atmosphere interact with the cool disk material and produce a warm ( 80 keV) surface layer, where soft photons from the cool disk are Comptonized. We have considered two cases in detail: a disk around an $8 M_{\odot}$ black hole (galactic BHC case), and an AGN case with an $8 \times 10^{6} M_{\odot}$ black hole.

We find that the response of the cool disk to proton illumination depends on its optical thickness. At sufficient thickness $(\tau \gtrsim 1.5)$ the cool disk is able to effectively reprocess incoming energy flux into soft photons. Here, a two-layer structure develops, consisting of a warm surface layer of depth $\tau \sim 1$ on top of a cool disk. At lower total optical depth, the soft photon emissivity is insufficient to cool the disk and the disk heats up, by the proton energy flux, to an approximately isothermal layer of intermediate (a few $100 \mathrm{keV}$ ) temperatures. We call these cases warm disks: their temperature is higher than an accretion disk radiating as a black-body, but still much lower than the virial temperature.

In the optically thick case, the energy balance between the warm surface layer and the cool disk is like that in the Haardt-Maraschi $(1991,1993)$ model of a corona over a cool disk. For geometrical reasons about half of the Comptonized photons is reprocessed by the cool disk, the other escapes. The Compton $y$-parameter adjusts such that the amplification factor matches this requirement. This balance regulates the temperature of the layer, while its optical depth is determined by the penetration depth of the protons. The balance is strongly self-regulating: the temperature and optical depth of the warm layer depend little (within limits) on temperature and energy flux of the incident protons, so that also the shape of the emergent X-ray spectrum varies little (see Haardt \& Maraschi 1993, and Paper I for a more detailed discussion).

We find that the existence of these proton-heated surface layers is limited to the innermost regions $r \lesssim 50$ of the disk. At larger distances from the hole, the optical depth of the warm layer drops rapidly, and its contribution to the spectrum becomes small.

For our BHC case the temperature and optical depth of the warm layer are in the right range to produce spectra with the main features of black hole binaries in their hard states. In the AGN case, the Comptonization is weaker, and the hard spectral component somewhat steeper (photon index around 2.6) than in the BHC case (index around 2.2). In both the BHC and AGN cases the spectra cut off around $200 \mathrm{keV}$. The maximum distance from the hole where a significant warm layer is formed is somewhat smaller in the AGN case $\left(R_{\max } / R_{\mathrm{S}} \approx 20\right)$ than in the BHC case $\left(R_{\max } / R_{\mathrm{S}} \approx 50\right)$. This is due to the lower energy flux per unit of surface area, and the 
reduced penetration depths in the cooler AGN disks (see also Deufel et al. 2001).

Near the inner edge of a cool accretion disk (at the distance $R_{\mathrm{i}}$ from the hole), its optical depth becomes small (in the thin disk limit, it varies by the well known factor $\left.f=\left[1-\left(R_{\mathrm{i}} / R\right)^{1 / 2}\right]\right)$. Though the optical depth of a cool accretion disk is generally substantial at the accretion rates inferred for BHC and AGN, there is always a region of low optical depth near the inner edge. This is also where the disk would be exposed to the largest proton energy flux from an ADAF or corona. At optical depths $\tau_{1 / 2} \lesssim 1.5$ we find that an initially cool disk heats up to a new equilibrium with nearly uniform temperatures of a few $100 \mathrm{keV}$ (Fig. 5). The transition takes place in a time of the order of the dynamical time scale $1 / \Omega$. Initially, the protons are stopped in the cool layers before reaching the midplane. Due to the thermal expansion of the plasma the electron densities drop, and as a consequence the proton heating finally overcomes the cooling by bremsstrahlung $\left(\sim n_{\mathrm{e}}^{2}\right)$. A new equilibrium is found when pair production sets in. The pairs add to the optical depth of the layer and increase the radiative loss of the plasma (by Comptonization) until it matches the input by proton heating.

Our solutions are local equilibria for conditions at a given distance from the hole. In disks in which the optical depth is low $(\lesssim 1)$ near the inner edge but at least a few at larger distance, proton heating would produce our warm disk structure of approximately uniform temperature in a (possibly narrow) zone near the inner edge. Further out it would instead produce a warm surface layer overlying the cool disk. Both regions would contribute to the overall X-ray spectrum.

Since the energy flux by proton heating is highest near the inner edge, the warm disk component at that location could contribute significantly to the overall spectrum even if its radial extent is limited. In our solutions, it would add a very flat component (photon index around 1, up to about $1 \mathrm{MeV}$, Fig. 4), so the overall spectral index could be significantly less than 2, as observed in some of the hardest spectra. The warm surface layers over the cool disk do not have such flat spectra (Fig. 2), and alone can not explain the hardest observed spectra (with indices around 1.5). The combination has the potential to explain the entire range of observed hard spectra, but for a quantitative result the radial structure of a proton-illuminated disk needs to be treated in more detail.

In this context we note that our warm disk solutions ignore a potentially important cooling mechanism, namely Compton cooling by soft photons from a cool disk. Though there are no cool regions internally in the warm disk, the nearby cool disk at larger radii produces a soft photon flux. A fraction of this flux could travel radially, illuminating the warm disk region. The flux of such soft photons is probably small, since the disk is thin at all radii and the radial optical depths therefore large. For quantitative assessment a two-dimensional radiative transfer model has to be developed, which is outside the scope of the present treatment.
The warm disk solution has a combination of temperature and optical depth just in the region where a normal, internally heated disk cannot exist. In such disks, the $(T, \tau)$ combination characterizing our warm disks would lie on the SLE (Shapiro-Lightman-Eardley) branch of accretion flows, which is thermal/viscously unstable. The combination is quite stable in our warm disk solution precisely because it is not heated internally but externally by the incident protons. In this context it is interesting that model fits of low/hard states in terms of stationary ADAF models sometimes yield parameter combinations on the unstable SLE branch (for example in XTE 1118 +480 as analyzed by Frontera et al. 2001). We interpret this as a strong indication for the existence of our warm disk solutions.

The physics of the warm disk solutions presented here has further interesting consequences. In anticipation of results to be given in more detail in a future paper, we note here that at the temperatures and densities in our warm disks, the time scale for establishing thermal equilibrium between the electrons and the protons in the disk (not the illuminating protons) is not short compared to the dynamical time scale any more. Any mechanism which now provides energy to the disk protons faster than they can exchange energy with the disk electrons would give rise to a heating of the disk protons and, depending on the temperature and density dependence of such a process, might lead to runaway heating of the protons. Two energy channels immediately come to mind: proton-proton interactions, i.e. heating of the disk protons by the penetrating external protons, and internal viscous heating according to standard disk theory. We have neglected these here, as their energetic contribution is negligible in our model (the main energy channel is from the incident protons to the radiation field, via the disk electrons). If such an instability exists, it might heat the disk protons sufficiently for the disk to expand and feed its mass into the surrounding ADAF.

Acknowledgements. This work was done in the research network "Accretion onto black holes, compact stars and proto stars" funded by the European Commission under contract number ERBFMRX-CT98-0195.

\section{References}

Aharonian, F., \& Sunyaev, R. 1984, MNRAS, 210, 257

Alme, M. L., \& Wilson, J. R. 1973, ApJ, 186, 1015

Chandrasekhar, S. 1942, Principles of Stellar Dynamics (University of Chicago Press, 1992.)

Deufel, B., Dullemond, C. P., \& Spruit, H. C. 2001, A\&A, 377, 955

Deufel, B., \& Spruit, H. C. 2000, A\&A, 362, 1 (Paper I)

di Matteo, T., Celotti, A., \& Fabian, A. C. 1999, MNRAS, 304, 809

Dove, J. B., Wilms, J., Maisack, M., \& Begelman, M. C. 1997, ApJ, 487, 759

Esin, A. A., McClintock, J. E., \& Narayan, R. 1997, ApJ, 489, 865 
Haardt, F., \& Maraschi, L. 1991, ApJ, 380, L51

Haardt, F., \& Maraschi, L. 1993, ApJ, 413, 507

Haardt, F., Maraschi, L., \& Ghisellini, G. 1994, ApJ, 432, L95

Haardt, F., Maraschi, L., \& Ghisellini, G.1997, ApJ, 476, 620

Jauch, J. M., \& Rohrlich, F. 1976, The theory of photons and electrons, The relativistic quantum field theory of charged particles with spin one-half (Texts and Monographs in Physics, New York: Springer, 1976, 2nd ed.)

Martín, E., Rebolo, R., Casares, J., \& Charles, P. 1994a, ApJ, 435, 262

Martín, E., Spruit, H., \& van Paradijs, J. 1994b, A\&A, 291, 43

Nakamura, K., \& Osaki, Y. 1993, PASJ, 45, 775

Narayan, R., \& Yi, I. 1994, ApJ, 428, L13

Narayan, R., \& Yi, I. 1995a, ApJ, 444, 231

Narayan, R., \& Yi, I. 1995b, ApJ, 452, 710

Pozdnyakov, L. A., Sobel, I. M., \& Syunyaev, R. A. 1983, Astroph. and Space Phys. Res., 2, 189

Rees, M. J., Phinney, E. S., Begelman, M. C., \& Blandford, R. D. 1982, Nature, 295, 17
Ross, R. R., \& Fabian, A. C. 1993, MNRAS, 261, 74

Shakura, N. I., \& Sunyaev, R. A. 1973, A\&A, 24, 337

Shapiro, S. L., Lightman, A. P., \& Eardley, D. M. 1976, ApJ, 204,187

Spruit, H. C. 1997, in Lecture Notes in Physics (Berlin Springer Verlag), vol. 487, 67

Spruit, H. C., \& Haardt, F. 2000, MNRAS, 315, 751

Stepney, S., \& Guilbert, P. W. 1983, MNRAS, 204, 1269

Sunyaev, R. A., \& Titarchuk, L. G. 1980, A\&A, 86, 121

Svensson, R. 1982, ApJ, 258, 335

Svensson, R., \& Zdziarski, A. A. 1994, ApJ, 436, 599

Turolla, R., Zampieri, L., Colpi, M., \& Treves, A. 1994, ApJ, 426, L35

Zampieri, L., Turolla, R., Zane, S., \& Treves, A. 1995, ApJ, 439,849

Zane, S., Turolla, R., Zampieri, L., Colpi, M., \& Treves, A. 1995, ApJ, 451, 739

Zel'dovich, Y. B., \& Shakura, N. I. 1969, Soviet Astron.-AJ, 13, 175, ZS69 